\section{New Drug Approval}

\section{Sofosbuvir, velpatasvir and voxilaprevir}

Date of Approval: July 18, 2017

Treatment for: Chronic Hepatitis C

Vosevi (sofosbuvir, velpatasvir and voxilaprevir or SOF/VEL/VOX) is a fixed-dose combination of a nucleotide analogue NS5B polymerase inhibitor (SOF), a pangenotypic NS5A inhibitor (VEL), and a pangenotypic NS3/4A protease inhibitor (VOX) for the treatment of genotype 1-6 chronic hepatitis $\mathrm{C}$ virus (HCV) infection.

\section{Neratinib}

Date of Approval: July 17, 2017

Treatment for: Breast Cancer -- Adjuvant

Company: Puma Biotechnology, Inc.

The U.S. Food and Drug Administration (FDA) has approved Nerlynx (neratinib), a once-daily oral tyrosine kinase inhibitor for the extended adjuvant treatment of adult patients with early stage HER2overexpressed/amplified breast cancer, following adjuvant trastuzumab-based therapy.

\section{Guselkumab}

Date of Approval: July 13, 2017

Treatment for: Plaque Psoriasis

The U.S. Food and Drug Administration (FDA) has approved Tremfya (guselkumab), an interleukin-23 blocker for the treatment of adult patients with moderate-to-severe plaque psoriasis who are candidates for systemic therapy or phototherapy.

\section{L-glutamine}

Date of Approval: July 7, 2017

Treatment for: Sickle Cell Anemia

Endari (L-glutamine) is orally-administered pharmaceutical grade L-glutamine (PGLG), an amino acid formulation to relieve pain, swelling and other complications of sickle cell anemia.

\section{Triptorelin}

Date of Approval: June 29, 2017

Treatment for: Precocious Puberty
Triptodur (triptorelin) is a gonadotropin releasing hormone (GnRH) agonist indicated for the treatment of pediatric patients with central precocious puberty.

\section{Betrixaban}

Date of Approval: June 23, 2017

Treatment for: Prevention of Venous

\section{Thromboembolism}

Bevyxxa (betrixaban) is an oral, once-daily Factor Xa inhibitor anticoagulant for the extended-duration prophylaxis of venous thromboembolism (VTE) in atrisk adult patients hospitalized for an acute medical illness.

C1 esterase inhibitor (human) Subcutaneous Injection

Date of Approval: June 22, 2017

Treatment for: Hereditary Angioedema

Haegarda (C1 esterase inhibitor (human)) is a lowvolume subcutaneous (SC) C1-esterase inhibitor (C1INH) replacement therapy to prevent Hereditary Angioedema (HAE) attacks.

\section{Rituximab and hyaluronidase}

Date of Approval: June 22, 2017

Treatment for: Follicular Lymphoma; Diffuse Large B-Cell Lymphoma; Chronic Lymphocytic Leukemia Rituxan Hycela (rituximab and hyaluronidase human) is a subcutaneous monoclonal antibody and hyaluronidase human formulation for the treatment of adult patients with follicular lymphoma, diffuse large B-cell lymphoma (DLBCL), and chronic lymphocytic leukemia (CLL).

\section{Delafloxacin}

Date of Approval: June 19, 2017

Treatment for: Skin and Structure Infection

Baxdela (delafloxacin) is a fluoroquinolone antibacterial indicated for the treatment of acute bacterial skin and skin structure infections (ABSSSI) caused by designated susceptible bacteria.

Source: drugs.com

Information collected and compiled by:

Mr. Md. Akbar Hossain

Assistant Professor

Department of Pharmacy

ASA University, Dhaka 